\title{
CLÚSTER VIRTUAL: NUEVA ALTERNATIVA A LA COMPETITIVIDAD EFICAZ EN LAS EMPRESAS
}

\author{
VIRTUAL CLUSTER: NEW ALTERNATIVE TO EFFECTIVE \\ COMPETITIVENESS IN ENTERPRISES
}

\section{CLUSTER VIRTUAL: NOVA ALTERNATIVA À COMPETITIVIDADE EFICAZ NAS EMPRESAS}

\section{FERNÁNDEZ HURTADO_Saúl Rick, CASTILLO TRIANA_Diego, MARTÍNEZZ MARTÍNEZ_Luz Ángela}

Postdoctor en Globalización y Desarrollo Regional, Center for Global Studies, Shanghai University. Profesor Universidad Santiago de Cali. Email: saulrick@163.com, Colombia.

Master en Educación Superior, Universidad Santiago de Cali; Profesor de la Universidad San Martin, Cali - Colombia; Profesor Universidad del Valle. Email: diego.castillo01@gmail.com, Colombia.

Profesional en Ingeniería Industrial, Universidad Autónoma de Occidente, Departamento de Operaciones y Sistemas, Email: luz_angela.martinez@uao. edu.co, Colombia.

Recibido: 8 de febrero de 2017 Aprobación definitiva: 23 de mayo de 2018

DOI: http://dx.doi.org/10.22267/rtend.181901.92

\begin{abstract}
RESUMEN
El artículo estudia y analiza la gestión del networking empresarial y la creación de clústeres virtuales de empresas. El propósito principal de la investigación, es indagar acerca de la posibilidad de crear una plataforma de clúster virtual innovador; que pueda ser visto como una innovación práctica que fomente el crecimiento económico, a partir de lo local, mejorando la competitividad empresarial.
\end{abstract}


Durante la investigación, se profundizó en los conceptos de la generación de networking, el cual es una plataforma para mejorar el trabajo colaborativo entre organizaciones y permite consolidar la conformación de clústeres empresariales. Igualmente, se identificaron los beneficios e inconvenientes de crear un clúster virtual. El escenario de clúster virtual permite alcanzar una mayor eficiencia en el logro de objetivos individuales y colectivos; su mayor impacto se observa en la reducción de los costos de transacción internacional y en la ampliación de las cuotas de mercados, motivando una colaboración efectiva de los recursos por parte de cada miembro del clúster. La sinergia del networking y el clúster virtual, favorece la creación de nuevos modelos de negocio innovadores, fortalece las posibilidades de adquirir nuevas tecnologías y genera nuevos conocimientos técnicos, con lo cual se abren nuevas oportunidades de crecimiento a través de clústeres virtuales y empresas innovadoras.

Palabras claves: Clúster virtual, networking, cooperación, China, tecnología.

JEL: P13

\section{ABSTRACT}

The articles studies and analyzes the management of business networking and the creation of company's virtual clusters. The main purpose of the research is to study the possibility to build an innovative virtual cluster platform; that can be seeing as a practical innovation that fosters economic grow, from the local, improving competitive businesses.

During the research, delved into the networking generation concepts, which is a platform used to improve collaborative work among organizations and allow to consolidate business cluster. In addition, the benefits and disadvantages of building virtual cluster were identified. A virtual cluster scenario enables to achieve greater efficiency in the achievement of individual and collective goals; its major impact its observed in the reduction of international transaction costs and the expansion of markets quotas, encouraging effective collaboration of resources by each member of the cluster. The synergy of networking 
and the virtual cluster, it make a benefit to build an innovative business model, strengthens the possibilities of acquiring new technologies and know-how, which open new growth opportunities through virtual cluster and innovative companies.

Keywords: Virtual cluster, networking, cooperation, China, technology. JEL: P13

\section{RESUMO}

Os artigos estudam e analisam o gerenciamento de redes de negócios e a criação de clusters virtuais da empresa. O principal objetivo da pesquisa é estudar a possibilidade de construir um plataforma de cluster virtual inovador; isso pode ser visto como uma inovação prática que promove o crescimento econômico, do local, melhorando negócios competitivos.

Durante a pesquisa, mergulhou nos conceitos de geração de rede, que é um plataforma usado para melhorar o trabalho colaborativo entre organizações e permitir a consolidação do cluster de negócios. Além disso, os benefícios e desvantagens da construção de cluster virtual foram identificados. Um cenário de cluster virtual permite alcançar maior eficiência no alcance de objetivos individuais e coletivos; seu maior impacto é observado na redução dos custos de transação internacional e na expansão das cotas de mercado, incentivando a colaboração efetiva de recursos por cada membro do cluster. A sinergia da rede e do cluster virtual, faz um benefício para construir um modelo de negócio inovador, fortalece as possibilidades de adquirir novas tecnologias e know-how, que abrem novas oportunidades de crescimento através de cluster virtual e empresas inovadoras.

Palavras-chave: Cluster virtual, redes, cooperação, China, tecnologia. JEL: P13 


\section{INTRODUCCIÓN}

\section{Clúster Industrial y sus políticas}

En los países desarrollados, los entornos de la industria son sumamente importantes en las economías, las externalidades hacen parte de los planes que sustentan la estrategia de crecimiento económico y desarrollo social en estos países. Así por ejemplo: China tiene como variable de desarrollo global la innovación en sus diversos entornos productivos (Zhang, Zheng, Song, Deng, \& He, 2015; Tian et al., 2017), generando impactos positivos en su economía. Las empresas multinacionales en China y otras del continente asiático hacen parte del networking de innovación y desarrollo industrial, por eso la economía China al incluir la innovación en forma transversal en su política económica se atempera a este importante requisito contemporáneo como lo han manifestado por Dai \& Zhang, (2017).

Según Fernández, (2013), los enfoques de Clúster en la dinámica empresarial tienen un papel cada vez más notorio en la generación de riqueza. Fernández, (2013) menciona que el Networking de las empresas, con proximidad geográfica, da mejores resultados si está integrado por unidades económicas de igual o similar grado de desarrollo, consolidando un clúster para interactuar con mayor capacidad productiva en los mercados.

En la dinámica de los Clúster a nivel geográfico, como por ejemplo: los existentes en países de la Comunidad de Estados Americanos y Caribeños (CELAC), se observa que estos actúan con diversas empresas (pequeñas, medianas y grandes), conformando un networking de acuerdo a su tipo de complementariedad en su producción (S., 2013). Y, por ello, al gestionarse un networking empresarial, se forman clústeres productivos de bienes y servicios, para satisfacer las nuevas necesidades de los consumidores en los distintos mercados como lo ha referido (Terho \& Jalkala, 2017) y (Ceric, D'Alessandro, Soutar, \& Johnson, 2016) en su estudio. Las empresas dejan su entorno de confort y migran hacia la cooperación e ingreso a mercados que no estaban previstos en sus agendas de expansión, dado que su producción y sus 
capacidades financieras no lo permitían. La férrea competencia que orientaba parte de la estrategia empresarial cambia en los escenarios internacionales por la cooperación mutua que se ha integrado en el clúster. El citado, Fernández, (2013) manifiesta en su estudio que los gobiernos durante la conformación de clústeres industriales de empresas, tienen la responsabilidad de facilitar el funcionamiento de asociaciones públicoprivadas dentro de los acuerdos que poseen con otros gobiernos. Para el efecto, es recomendable utilizar las ventajas que incorporan los tratados bilaterales, que al implementarlas, facilitan y agilizan los procesos en la conformación de networking, y aumentan la eficacia de los Clústeres en la conquista de mercados potenciales.

\section{MARCO TEÓRICO}

\section{Enfoque del Clúster: beneficios y preocupaciones}

El surgimiento de Clústeres como estrategia en el desarrollo económico e industrial, genera diversidad dentro de los mercados donde los consumidores tienen la capacidad de satisfacer gustos por la multiplicidad de productos internacionales y las empresas incrementan su potencial productivo al ampliar su portafolio de bienes y servicios (Kamoche, Pang, \& Wong, 2011; Bajzikova, Sajgalikova, Wojcak, \& Polakova, 2016; Rick, Hurtado, Ángela, \& Martínez, (2018). La integración y la cooperación que brinda un ambiente de relaciones con beneficios mutuos, al interior de los clústeres de empresas, presentan los siguientes beneficios como lo destacan los autores en la figura 1:

1. Desarrolla los efectos de cooperación empresarial

2. Las empresas aumentan los entornos de innovación (Ej. China)

3. Se incrementa la empleabilidad

4. Mejora el crecimiento económico en los países

5. Genera una alta dinámica en productividad, favorece el crecimiento en las transacciones internacionales

6. Incrementa la capacidad de inversión conjunta entre empresas 
7. Fortalece la capacidad de pagos de acuerdo a la integración empresarial

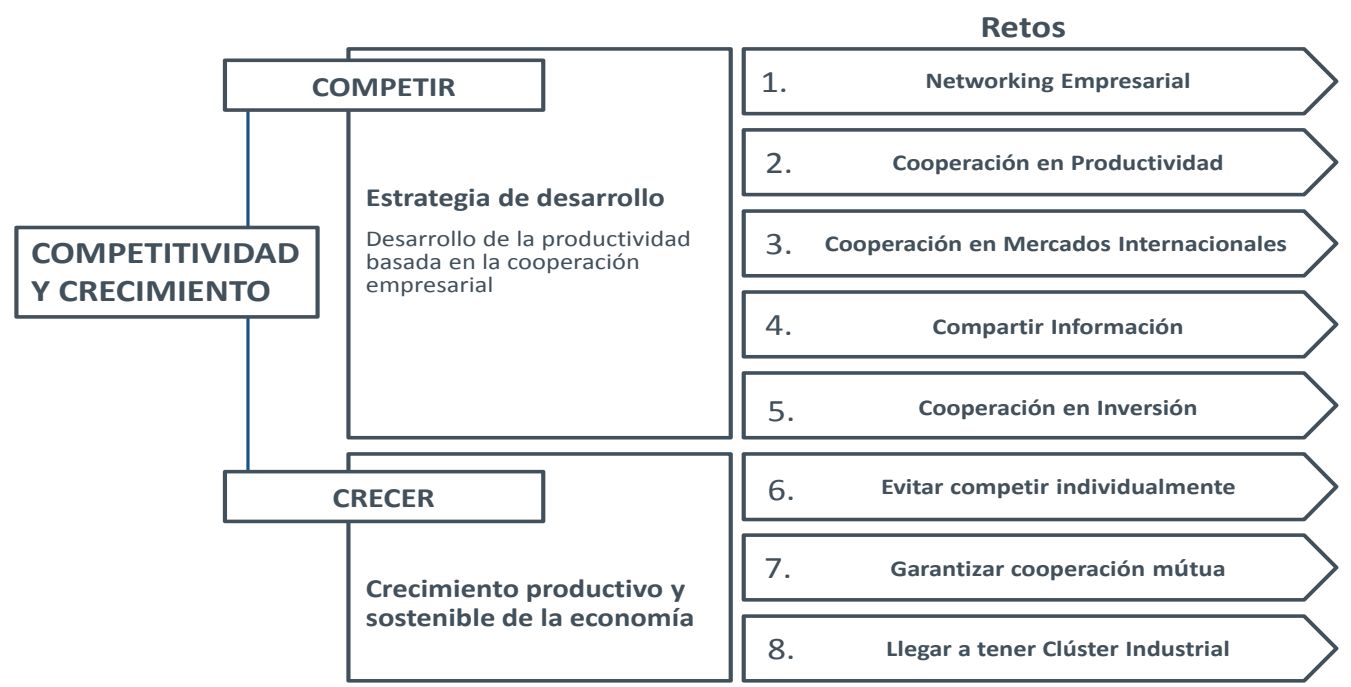

\section{Figura 1}

Beneficios en Integración de Clústeres

Fuente: Elaboración por los autores (2017).

En el proceso de implementación de los Clústeres empresariales se detectan algunos inconvenientes, esto obedece a la carencia de gestión del networking de empresas con alta capacidad productiva, la falta de visión de integración de mercados y el retraso en ampliar la zona de confort de lo nacional a lo internacional. Por lo tanto, para superar las debilidades anteriores, se requiere que las empresas tengan mayor confianza entre sí (S. Rick, 2013; Belkadi \& Bernard, 2015). Los principales inconvenientes, son:

- El desarrollo de Clústeres ha tenido una falla en su implementación, hay muchas empresas disimiles en cada industria.

- Los enfoques de trabajo grupal e individual en la implementación de clústeres se debe coordinar más acertadamente, buscando una mejor participación conjunta de las empresas asociadas en la reducción de costos y riesgos. 
- Durante el desarrollo de los clústeres empresariales, la variable confianza es un término complejo en las empresas pertenecientes a las industrias. Cuando el desarrollo de los clústeres es generado mediante el networking de empresas, la falta de confianza desplaza los beneficios mutuos que tendrían las compañías en los mercados (ver Figura 1).

- La falta de un proceso de comunicación asertiva en la implementación de clústeres y en la gestión de networking empresarial (Fernández, 2013; Negruşa, Rus, \& Sofică, 2014; Beckwith \& Ceriani, 2015; Al-Anazi, AlMahmoud, \& Al-Turaiki, 2016) no ayuda a la transparencia y cooperación de información en todo nivel (Ej. Información de mercados), por lo tanto no se genera una competencia leal.

Durante la implementación de clústeres yal establecer los temas prioritarios, las empresas tienen la potestad de seleccionar y evaluar sus relaciones con otras mediante el networking que estas desarrollan. Los autores resaltan que la capacidad de información que cada una provee mediante la confianza generada en los procesos de cooperación mutua mejora la participación de las industrias nacionales en los mercados específicos.

Durante el estudio se evidenció que los clústeres son mucho más dinámicos en los sectores económicos donde las prácticas del networking son más frecuentes y articuladas por las empresas que interactúan en el clúster.

Esta dinámica está relacionada con la priorización del desarrollo de cada empresa y su creación de ventajas compartidas durante la evolución de una red empresarial. Esta integración es más fuerte cuando las empresas transnacionales tienen la visión de construir vínculos intrarregionales (Oh \& Rugman, 2012; Ogrean, 2013; Kasper, Lehrer, Mühlbacher, \& Müller, 2013) en busca de ampliar sus zonas de confort por fuera de sus fronteras. Así las cosas, una integración bajo el esquema de clúster puede ser vista como un Joint Venture, así lo estudió (Li \& Parboteeah, 2015), sin embargo, la realidad indica que no puede ser considerada como tal, por la simple razón de que el clúster es más una cooperación mutua entre las partes, en busca de ampliar sus mercados en este caso 
de investigación (Lee, Rosenthal, Veld, \& Veld-Merkoulova, 2015), y no cuentan con metas comunes sino más bien individuales, como también con objetivos y proyectos autónomos, compartiendo múltiples ámbitos de información y áreas de cooperación. Por otra parte, la integración y la cooperación industrial causan interpretaciones diversas como lo manifiesta (Kasper et al., 2013; Fernández, 2013), por lo que las empresas seguirán llevando a cabo sus actividades de cooperación y no existen mecanismos establecidos y modos de interacción preestablecidos. Esto hace que cada empresa al interior del clúster se rija por su propia cultura organizacional y la responsabilidad en las actividades compartidas no son conjuntas sino individuales.

Igualmente, existen combinaciones en el desarrollo de negocios formadas por mutuo acuerdo y beneficios, que presentan objetivos con alta efectividad, que no son formalmente identificados como clústeres (Fernández, 2013), pero que realmente requieren realizar una integración de recursos de cooperación para ingresar a mercados donde individualmente no podrían hacerlo por la carencia de recursos tales como: monetarios, conocimientos, networking; esto obedece a la necesidad de obtener apoyo externo como también de acceder a las plataformas que los gobiernos han creado tiempo atrás (Rick et al., 2018). Por lo anterior, no se pueden presentar confusiones acerca de la generación de clústeres industriales y los procesos de cooperación e integración empresarial (Fernandez, 2013); si se tiene en cuenta que la conformación de un clúster es identificable por las acciones colectivas de un grupo y en la toma de decisiones comunes en su desarrollo empresarial (ver Figura 4), como por ejemplo la conquista de mercados internacionales.

\section{DISCUSIÓN}

\section{Clúster de empresas virtuales y productivas}

Los clústeres deben ser efectivos y deben impulsar el desarrollo y cumplimiento de los objetivos de cada empresa participante. El networking es un catalizador de estos propósitos. Las empresas son creadas con la visión de ampliar sus mercados y están orientadas a reducir los costos asociados a las actividades productivas y sus entornos logísticos. Por otro lado, se forman con la intención de mejorar la colaboración idónea 
entre empresas de un mismo sector productivo (Cassiman \& Golovko, 2011; KjÆEr, 2015), obteniendo ventajas competitivas y contribuyendo a la transformación creativa de las empresas en su evolución hacia un clúster maduro. La integración colectiva y la circulación irrestricta de información y conocimiento potencializan la innovación necesaria para responder a los cambios que son requeridos en los diferentes entornos empresariales y sociales. Como ha sido discutido por los autores, el mundo cambiante nos exige desarrollar nuevos modelos de producción y de mercadeo, acordes con el vertiginoso cambio tecnológico y de las tecnologías de la informaci ón (Whiffen et al., 2016; Uribe-Pérez \& Pous, 2017) y las comunicaciones, (TIC'S). El hecho de coexistir empresas poco dinámicas y otras adelantadas en su desarrollo, perjudica el sector económico obteniendo un desplazamiento hacia atrás (Gurkan Inan \& Bititci, 2015; Krishnan \& Scullion, 2016). Por eso, los entornos de cambios sociales, políticos y empresariales nos hacen un llamado a generar frecuentemente métodos innovadores (Gurkan Inan \& Bititci, 2015; Henriques \& Catarino, 2016)para actuar con mayor rapidez y eficacia en las áreas funcionales de cada empresa. Por tal motivo, es vital aprender o más bien re-aprender de las capacidades innovadoras que posee cada empresa individual, lo que permite crear y tener una ventaja colectiva y sistemática del clúster como un todo. Ese proceso fomenta mecanismos que les permite interactuar con ventaja competitiva en los mercados, por eso la formación e implementación de clústeres virtuales y productivos puede llegar a ser un proceso creativo e innovador como se ilustra en la Figura 2. De hecho, lo es. (Alexander, Tatiana, \& Svetlana, 2013; Shin, Lee, Kim, \& Rhim, 2015).

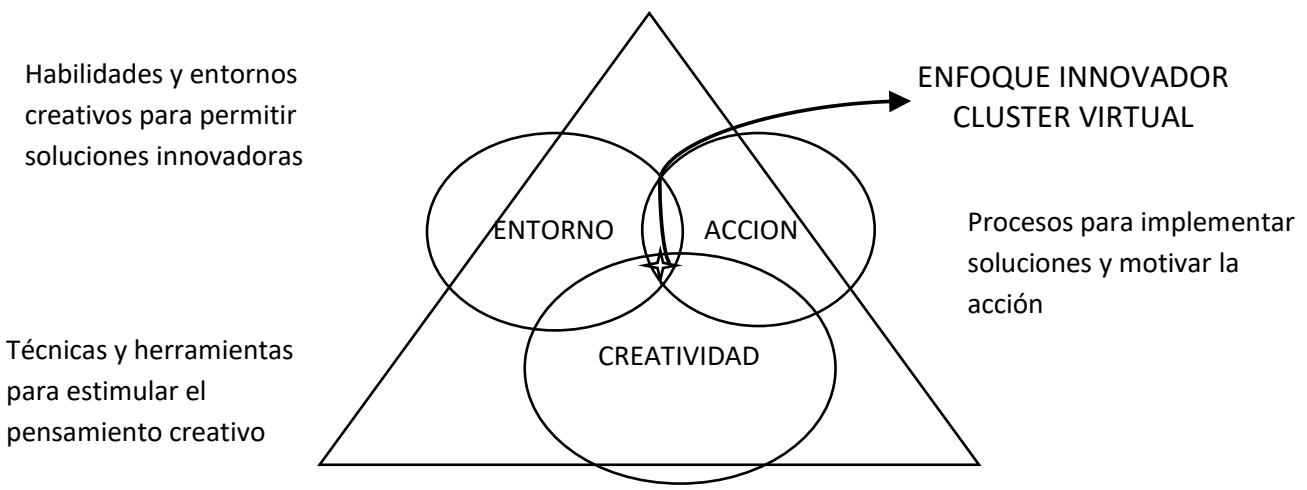

\section{Figura 2}

Modelo Clúster Virtual y Productivo

Fuente: Elaboración por los autores (2017) 
El clúster virtual y productivo en donde está la estructura tradicional se pueden ver con el mismo propósito, ya que cuentan con la misma capacidad y potencial en favor de los objetivos de mercado (Fernández, 2014), que en ambos casos son similares. Esa capacidad es vista en diversas dimensiones empresariales y formas de intercambio y cooperación centrada en una plataforma de cooperación mutua. Su funcionamiento permite cumplir los objetivos individuales de cada parte y simplemente cada integrante logra sus ventajas competitivas optimizando la producción y proporcionando altos beneficios dentro del networking (Kasper et al., 2013; Beckwith \& Ceriani, 2015).

El networking empresarial en el ámbito de la cooperación virtual y productivo, tienen la potestad de compartir sus capacidades y recursos en busca de los objetivos empresariales y de igual forma brindan beneficios a otras empresas que integran el clúster virtual como se observa en la Figura 3 (Fang, 2012; Shin et al., 2015). Esto permite lograr buenos resultados económicos y mayor eficiencia en los escenarios internacionales al emplear una dinámica competitiva como es destacado por el gobierno ruso (Becker \& Vasileva, 2017). Desde el punto de vista del consumidor, las empresas clusterizadas son percibidas como individuales (Lee et al., 2015; Beckwith \& Ceriani, 2015), logrando que el consumidor no entre en dilemas de altos costos por el síndrome de los intermediarios, el clúster virtual y productivo permite obtener información moderna y tecnologías de comunicación apropiadas, esto sin tener afectación comunicativa entre distintos países en la cadena de valor global.

Naturalmente el clúster virtual y productivo está integrado por empresas individuales, las cuales buscan objetivos propios. Estas empresas no tienen la responsabilidad de estrechar lazos como en el caso de joint venture, pero si tienen el compromiso de gestionar una buena comunicación al estar en diferentes lugares geográficos. (Lee et al., 2015).

El clúster virtual y productivo va más allá del intercambio de ventajas individuales, conforma una amplia red de cooperación entre sus miembros para conquistar a los consumidores externos. El operar con un solo método se puede convertir en un problema sino se estudian otros métodos descriptivos que incidan directamente en una plataforma empresarial en el que se desenvuelve el clúster. De tal manera que no es 
aconsejable utilizar una sola metodología en busca de nuevos mercados como lo resalta (Fernández, 2013). Los constantes cambios sociales, económicos y políticos que viven permanentemente los países obligan a combinar las acciones del clúster con el fin de realizar cambios que generen conocimientos y tecnologías en beneficio del entorno virtual y productivo. Las empresas que actúan en un contexto virtual y productivo, serán el núcleo del clúster (Fernández, 2013; Romero \& Noran, 2015).

Como se puede apreciar en la Figura 3, el networking de empresas con habilidades virtuales y productivas precisa al apoyo gubernamental para usufructuar las relaciones bilaterales que cada país ha acordado:

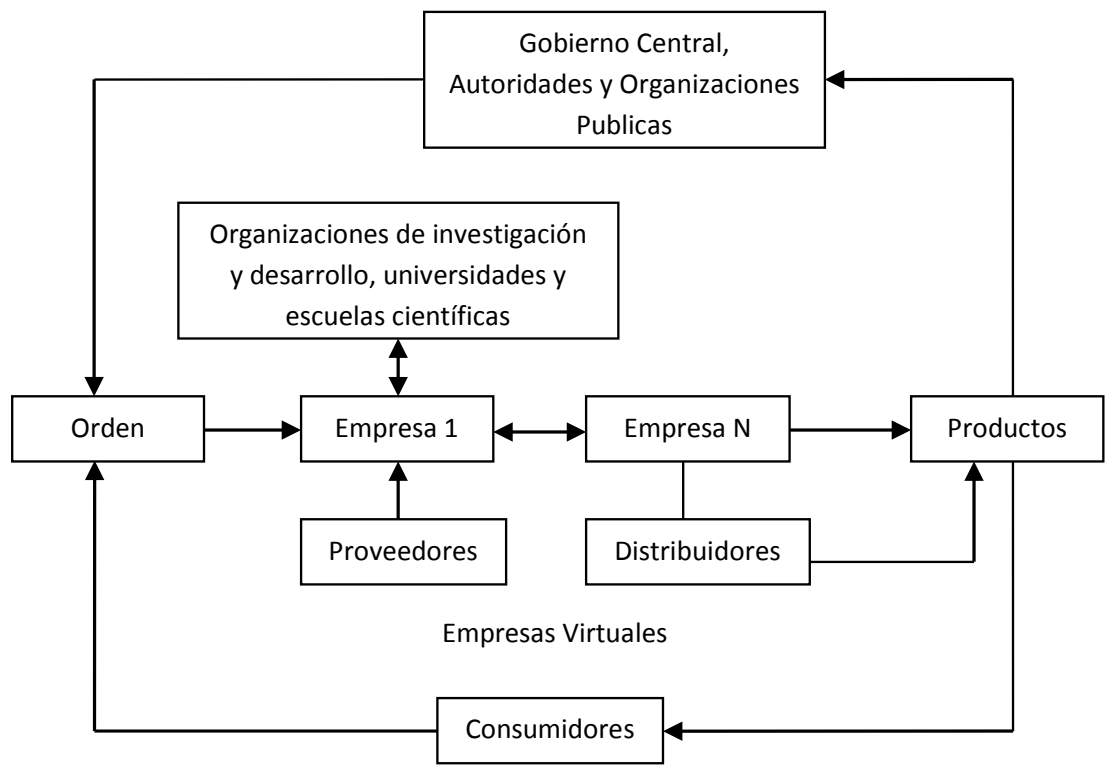

\section{Figura 3}

Clúster Virtual y Productivo en las Empresas

Fuente: Elaboración por los autores (2017).

Los procesos al interior del clúster virtual y productivo generan impactos innovadores en sus empresas, que tienen como objetivo dinamizar sus plataformas de ingresos y racionalizar los egresos. Al estudiar un clúster virtual y productivo de empresas que trabajan por obtener una misión común, se aprecia que buscan alcanzar un alto rendimiento empresarial generando valor para cada una de ellas y los consumidores se ven favorecidos al contar con diversas opciones de consumo. 
Así, cuando las empresas del clúster buscan sus objetivos individuales y cooperan en logros comunes de la aglomeración industrial, el mejoramiento del sector económico es notorio. Esto permite mejores beneficios económicos y más participación en el mercado (Kasper et al., 2013; Beckwith \& Ceriani, 2015); las empresas que no han elevado sus niveles de competitividad, lo harán con mayor tacto y precaución, debido a la cooperación con múltiples empresas con objetivos similares. Durante la generación del clúster, el $80 \%$ del éxito competitivo de cada empresa depende del aporte de su pasión (Fernández, 2013) y de la combinación de los puntos siguientes:

1. Intercambio con consumidores, en donde se realizan actividades de marketing, eventos y generación de bases de datos.

2. Intercambio con productores, incluyendo fuentes de materias primas y su cadena de abastecimiento

3. Adquisición de nuevas tecnologías que dinamicen actividades innovadoras.

4. Cooperación de las entidades gubernamentales, incluyendo la colaboración que brindan los tratados bilaterales, que generan beneficios en la actividad empresarial.

En el clúster virtual y productivo se tiene la ventaja de forjar innovación, a través de:

- Coordinar habilidades logrando objetivos individuales y proyectos empresariales comunes

1. Cada miembro del Clúster Virtual y Productivo, debe orientar gran parte de sus esfuerzos en gestionar acciones empresariales eficientes y aprovechar sus habilidades internacionales de negociación. En este modelo las organizaciones que solo buscan objetivos individuales pueden causar desavenencias con las demás del clúster, por lo que deben apropiarse de una nueva cultura de negociación colectiva. 
2. En ese sentido ampliar la órbita de acción incrementa los riesgos. Las empresas dentro de un clúster virtual y productivo toman decisiones individuales y cuentan con el apoyo de la red, pero la toma de decisiones y riesgos a afrontar son propias. De esta forma, con el tiempo cada empresa tendrá la posibilidad de formar sus propias condiciones a través de la experiencia que obtiene de la red de clúster y de su singularidad en los procesos de negociación; buscando mejores beneficios y entrar a otros escenarios mundiales. (Fernández, 2013; Kasper et al., 2013; Beckwith \& Ceriani, 2015).

\section{- Reducción de costos durante los procesos operativos}

3. En el Siglo XXI los cambios en las actividades empresariales y en las unidades económicas que generan riqueza hacen que la cooperación se convierta en una fortaleza fundamental para el clúster virtual y sus canales productivos con el objetivo de ingresar a otros mercados. Esto facilita que cada empresa dentro de una plataforma de clúster virtual productivo tenga la oportunidad de reducir los costos tanto logísticos (Adele, Fouda, Romeo, Azizi, \& Fernández, 2014; Lee et al., 2015); como los asociados a los costos de conversión.

\section{- Disminución de los costos transaccionales (Ej. Información)}

4. Dentro de los entornos de negocios, la información es costosa y su transmisión tiene un valor elevado, al igual que su obtención. Para allegar la información que requiere la organización se necesita de múltiples actividades en los mercados externos donde tiene presencia cada empresa. La cooperación y la confianza creada por el trabajo del networking facilita la obtención de información útil para todos los miembros del clúster para el ingreso a los mercados objetivos. Conformar escenarios innovadores trae efectos positivos y beneficios para las empresas que trabajan dentro de un modelo de clúster virtuales productivos (ver Figura 2), al contar con el apoyo de las empresas agrupadas que trabajan objetivos comunes. 
- Disposición a participar en nuevos proyectos y capacidad de realizar operaciones empresariales con mayores recursos

5. La acción individual de las empresas que conforman el clúster hace que cada una aporte al proceso de innovación (Zhang et al., 2015; Dai \& Zhang, 2017) y a los demás procesos empresariales potenciando la capacidad organizacional conjunta. Esta sinergia mejora ventajas recíprocas entre los actores cuando penetran mercados internacionales; las inversiones en la cadena productiva igualmente favorece posicionarse en el mercado objetivo y satisfacer más allá las expectativas que tienen los consumidores en dichos mercados foráneos altamente competitivos según(Terho \& Jalkala, 2017) y (Ceric et al., 2016).

\section{- Beneficios en la reducción de las barreras fronterizas}

6. En el contexto del clúster virtual bajo el esquema de producción ante las empresas tienen mayor facilidad en gestionar sus actividades empresariales con mayor confianza en los escenarios internacionales. La posibilidad de brindar soluciones virtuales bajo la plataforma de canales de producción crea necesidades individuales, genera beneficios adicionales en la búsqueda de nuevos mercados. (Danis, De Clercq, \& Petricevic, 2011).

Las estrategias empresariales en los clústeres virtuales productivos tienden a hacer eficaces, por ejemplo, en la prestación de servicios entre diferentes mercados. En este campo las experiencias de las empresas transnacionales son destacables por tener acceso a información privilegiada para el análisis de mercado y el uso de plataformas tecnológicas avanzadas; les proporcionan un mayor cúmulo de información y conocimiento para la prestación de servicios innovadores que conllevan a que los consumidores finales adquieran de una forma fácil los productos ofertados; virtualmente enriquecen las cadenas productivas, convirtiendo estas en un escenario competitivo. 
En la cadena de valor global el networking ha tenido una dinámica progresiva y sus cambios tanto en integración mutua e individual alcanzan diferentes resultados y objetivos que se traducen en la generación de confianza en los consumidores y sus fidelización (Danis et al., 2011; S., 2013; Chang, Liu, \& Shen, 2017). Para lograr estos objetivos la producción debe ser limpia y sostenible como es destacada por los autores; lo que obliga a las empresas clusterizadas a innovar constantemente compartiendo ideas y soluciones radicales (Zhang et al., 2015; Dai \& Zhang, 2017). En ese trabajo colaborativo, los autores mencionan que el networking es una apuesta relevante para la integración de un clúster eficaz con múltiples iniciativas empresariales, como lo ilustra en la figura 4.

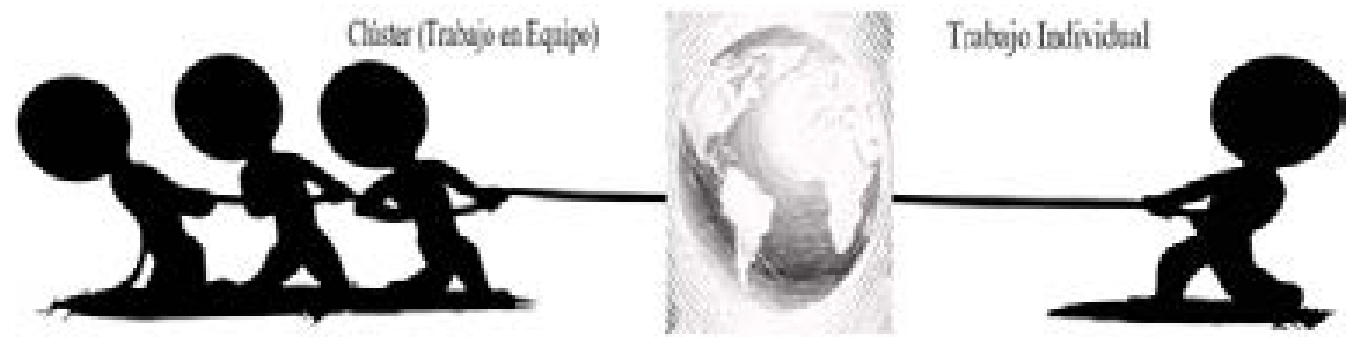

Figura 4

Clúster Eficaz

Fuente: Elaboración por los autores (2017).

La suma de las estrategias conjuntas en los clústeres virtuales originan sinergias y ventajas mutuas provocando un alto índice productivo, pero los objetivos individuales de las empresas clusterizadas virtualmente incrementan la productividad empresarial, definirán sus ventajas competitivas y por ende, sus costos de producción, administrativos y logísticos se reducirán, así manifiestan los autores. Este adelgazamiento en la estructura de costos perjudicará la generación de empleos en la próxima década; "pero no a todos los empresarios les preocupa los empleados/gente, las empresas no son instituciones de beneficencia". (Acs, Boardman, \& McNeely, 2013; González-Rodríguez, DíazFernández, \& Simonetti, 2015). Esto hace que los profesionales de hoy gestionen empresas innovadoras con diferentes valores agregados, generando cambios radicales en la sociedad que repercuten en la visión moderna de la economía y conducen a impulsar el crecimiento económico y un mejor desarrollo social. 
Los autores manifiestan que si se logra obtener en la plataforma del clúster virtual y productivo se obtendrá un escenario colaborativo en diversos recursos para una reducción de costos totales creando ventajas competitivas internacionales.

En China, el concepto de integración virtual y productiva se ha dinamizado durante décadas, la plataforma de negociación de networking empresarial es realizada en múltiples ocasiones con el propósito de dinamizar los negocios de diferentes sectores, al mismo tiempo, busca contribuir a que el networking ayude a crear empresas o plataformas empresariales innovadores con apuestas de valor diferente. De esta forma, este entorno facilita el intercambio de información y gestiona negocios con un direccionamiento particular, permitiendo ampliar el clúster virtual ante los puntos de vista productivos.

La revolución empresarial se ha caracterizado por los nuevos modelos de negocios basados en la innovación, siendo el consumidor quien tendrá los beneficios finales dado que la alta competitividad de las empresas permite que estas establezcan estrategias para optimizar los precios, ampliar los servicios, aumentar la calidad productiva, como también en pensar aún más en el consumidor final. (Negruşa et al., 2014; Lee et al., 2015).

Por ejemplo, los cambios realizados dentro de la revolución empresarial se presentan de acuerdo a la dificultad actual de llegar al consumidor, los consumidores tienen cada vez mayores opciones de compra y esto hace que la innovación para satisfacerlos aumente. El clúster permite que la integración en innovación sea mejor captada por los consumidores y estos no tengan múltiples opciones en la decisión de compra, por lo tanto distintas empresas al integrarse comparten una plataforma en común, con objetivos individuales, permitiendo un solo camino de consumo. Lo anterior da como resultado que las actividades generadas por la integración empresarial permitan que a nivel individual las empresas aumenten la capacidad de sus canales de consumo minimizando los riesgos que presentan los productos según su ciclo de vida. 
A nivel mundial muchos países no cuentan con este tipo de modelo de clúster virtual y productivo que ayuda a las empresas que trabajan bajo esta plataforma e igualmente beneficia a las regiones donde se localizan las aglomeraciones empresariales. Una de sus ventajas es la concentración de mano de obra especializada, además, los recursos serán más específicos por la ampliación de nuevas esferas de negocios.

Es relevante mencionar que la creación de networking obedece en la mayoría de los casos a grandes intereses y a necesidades comunes (Mäntymäki \& Riemer, 2016; Chang et al., 2017), cada miembro del clúster virtual y productivo al compartir sus opiniones individuales aumenta el capital social de la agrupación virtual; cada empresa dentro de la plataforma tiene el potencial de acceder al conocimiento y habilidades innovadoras brindadas y desarrolladas por los demás, esto debido a la transferencia que es brindada gracias al networking de las empresas en el clúster (S. Rick, 2014). Este tipo de modelo de negocios ha tenido crecimiento a nivel global, la globalización ha apoyado la dinámica comercial, por eso, los países firman acuerdos (Fernández Hurtado, 2013; Fernández, 2014) con el propósito de que las plataformas empresariales de cada país mejoren la capacidad y transformación de los mercados laborarles.

Así, las cosas, cada país al apoyar los distintos modelos de negocios, está en la obligación de mejorar las externalidades empresariales, tales como: Infraestructura, comunicaciones, servicios públicos, seguridad, entre otros, con el objetivo de que las empresas bajen los costos directos e indirectos y aumenten la productividad y competitividad, los autores afirman.

\section{CONCLUSIÓN}

El artículo estudia la conformación de una plataforma de clústeres virtuales y productivas destacando la participación y voluntad empresarial; durante esta investigación se analizaron algunas definiciones relevantes y enfoques de clúster de los expertos, (Fernández, 2013; Lee et al., 2015; Beckwith \& Ceriani, 2015; Dai \& Zhang, 2017). Igualmente, se observó 
que no obstante el trabajo colaborativo en ambas aglomeraciones empresariales, los enfoques de clúster industrial y clúster virtual bajo la productividad no tienen los mismos objetivos comunes sino individuales.

El tipo de método utilizado por empresas que participan en un clúster virtual productivo se fundamenta en la decisión de crear procesos de innovación que faciliten el acceso a los consumidores localizados en distintos lugares geográficos. Esa facilidad a la oferta productiva se da a través del trabajo colaborativo y el networking de empresas que poseen diferentes objetivos comerciales pero portafolios de productos similares. El networking al interior del clúster virtual productivo permite intercambiar información y conocimientos que favorece la ampliación de la cobertura del mercado objetivo convergiendo en mercados comunes.

Loanterior,fomentaunaampliacióndelascapacidadesorganizacionales en distintos niveles de recursos y conocimientos en el área de mercadeo mediante este modelo de clúster. La integración de empresas en el clúster virtual a nivel productivo mejora la productividad y competitividad, por ejemplo, compartiendo objetivos individuales y trabajando en equipo cada empresa tendrá mayor número de consumidores en su mercado objetivo (ver Figura 4).

Finalmente, se puede colegir que al reducir los costos de transacción se realiza un mayor número de operaciones comerciales a nivel local, nacional e internacional. Por último, es promisoria la formación de clústeres virtuales en los escenarios productivos; buscan mejoras en productividad y competitividad presentada por las empresas que conforman el clúster. 


\section{REFERENCIAS:}

(1) Acs, Z. J., Boardman, M. C., \& McNeely, C. L. (2013). The social value of productive entrepreneurship. Small Business Economics, 40(3), 785-796. http://doi.org/10.1007/s11187-011-9396-6

(2) Adele, R., Fouda, N., Romeo, N. D., Azizi, M., \& Fernandez, S. R. (2014). Port Logistics in West and Central Africa: A Strategic Development under Globalization. Open Journal of Applied Sciences, 4(4), 76-84. http://doi. org/10.4236/ojapps.2014.42009

(3) Al-Anazi, S., AlMahmoud, H., \& Al-Turaiki, I. (2016). Finding Similar Documents Using Different Clustering Techniques. Procedia Computer Science, 82, 28-34. http://doi.org/10.1016/j.procs.2016.04.005

(4) Alexander, B., Tatiana, K., \& Svetlana, U. (2013). Formation of Industrial Clusters Using Method of Virtual Enterprises. Procedia Economics and Finance, 5, 6872. http://doi.org/10.1016/S2212-5671(13)00011-7

(5) Bajzikova, L., Sajgalikova, H., Wojcak, E., \& Polakova, M. (2016). How Far is Contract and Employee Telework Implemented in SMEs and Large Enterprises? (Case of Slovakia). Procedia - Social and Behavioral Sciences, 235, 420-426. http://doi.org/10.1016/j.sbspro.2016.11.052

(6) Becker, U., \& Vasileva, A. (2017). Russia's political economy re-conceptualized: A changing hybrid of liberalism, statism and patrimonialism. Journal of Eurasian Studies, 8(1), 83-96. http://doi.org/10.1016/j.euras.2016.11.003

(7) Beckwith, E. J., \& Ceriani, M. F. (2015). Communication between circadian clusters: The key to a plastic network. FEBS Letters, 589(22), 3336-3342. http://doi.org/10.1016/j.febslet.2015.08.017

(8) Belkadi, F., \& Bernard, A. (2015). Trust-based patterns for the management of inter-enterprises collaborations in context of extended enterprise. IFACPapersOnLine, 48(3), 1186-1191. http://doi.org/10.1016/j.ifacol.2015.06.245

(9) Cassiman, B., \& Golovko, E. (2011). Innovation and internationalization through exports. J Int Bus Stud, 42(1), 56-75. 
(10) Ceric, A., D’Alessandro, S., Soutar, G., \& Johnson, L. (2016). Using blueprinting and benchmarking to identify marketing resources that help co-create customer value. Journal of Business Research, 69(12), 5653-5661. http://doi. org/10.1016/j.jbusres.2016.03.073

(11) Chang, S. E., Liu, A. Y., \& Shen, W. C. (2017). User trust in social networking services: A comparison of Facebook and Linkedln. Computers in Human Behavior, 69, 207-217. http://doi.org/10.1016/j.chb.2016.12.013

(12) Dai, R., \& Zhang, J. (2017). Green process innovation and differentiated pricing strategies with environmental concerns of South-North markets. Transportation Research Part E: Logistics and Transportation Review, 98, 132-150. http://doi. org/10.1016/j.tre.2016.12.009

(13) Danis, W. M., De Clercq, D., \& Petricevic, O. (2011). Are social networks more important for new business activity in emerging than developed economies? An empirical extension. International Business Review, 20(4), 394-408. http://doi. org/10.1016/j.ibusrev.2010.08.005

(14) Fang, T. (2012). All Business is Local: Why Place Matters More than Ever in a Global, Virtual World. John A. Quelch, Katherine E. Jocz, (c) 2012 Portfolio/ Penguin. 248 pp., ISBN 978-1-59184-465-5. International Business Review, 21(2), 307-309. http://doi.org/10.1016/j.ibusrev.2012.02.005

(15) Fernandez, S. R. (2013). Analytical, Descriptive and Exploratory Study: Challenges in the Implementation of Commercial Cluster Integration CELAC's Firms'. International Journal of Developing Societies, 2(3), 105-116. http://doi. org/10.11634/216817831504424

(16) Fernandez, S. R. (2014). Commercial Cluster Integration Initiative: To Develop International Diplomatic Commercial Relation Between Latin America \&quot; CELAC \&quot; and China. International J. Soc. Sci. \& Education, 4(2), 22234934.

(17) Fernandez Hurtado, S. R. (2013). Establishing An FTA Between China And Colombia Lowering Tariffs And Non-Tariff Barriers To Achieve Free Trade. LAP LAMBERT Academic Publishing.

(18) González-Rodríguez, M. R., Díaz-Fernández, M. C., \& Simonetti, B. (2015). The social, economic and environmental dimensions of corporate social responsibility: The role played by consumers and potential entrepreneurs. International Business Review, 24(5), 836-848. http://doi.org/10.1016/j. ibusrev.2015.03.002 
(19) Gurkan Inan, G., \& Bititci, U. S. (2015). Understanding organizational capabilities and dynamic capabilities in the context of micro enterprises: a research agenda. Procedia - Social and Behavioral Sciences, 210, 310-319. http://doi. org/10.1016/j.sbspro.2015.11.371

(20) Henriques, J., \& Catarino, J. (2016). Motivating towards energy efficiency in small and medium enterprises. Journal of Cleaner Production, 139, 42-50. http://doi.org/10.1016/j.jclepro.2016.08.026

(21) Kamoche, K., Pang, M., \& Wong, A. L. Y. (2011). Career Development and Knowledge Appropriation: A Genealogical Critique. Organization Studies, 32(12), 1665-1679. http://doi.org/10.1177/0170840611421249

(22) Kasper, H., Lehrer, M., Mühlbacher, J., \& Müller, B. (2013). On the different "worlds" of intra-organizational knowledge management: Understanding idiosyncratic variation in MNC cross-site knowledge-sharing practices. International Business Review, 22(1), 326-338. http://doi.org/10.1016/j.ibusrev.2012.05.001

(23) $\mathrm{Kj} /$ r, A. M. (2015). Political Settlements and Productive Sector Policies: Understanding Sector Differences in Uganda. World Development, 68, 230241. http://doi.org/10.1016/j.worlddev.2014.12.004

(24) Krishnan, T., \& Scullion, H. (2016). Talent management and dynamic view of talent in small and medium enterprises. Human Resource Management Review. http://doi.org/10.1016/j.hrmr.2016.10.003

(25) Lee, B., Rosenthal, L., Veld, C., \& Veld-Merkoulova, Y. (2015). Stock market expectations and risk aversion of individual investors. International Review of Financial Analysis, 40, 122-131. http://doi.org/10.1016/j.irfa.2015.05.011

(26) Li, C., \& Parboteeah, K. P. (2015). The effect of culture on the responsiveness of firms to mimetic forces: Imitative foreign joint venture entries into China, 19852003. Journal of World Business, 50(3), 465-476. http://doi.org/10.1016/j. jwb.2014.08.002

(27) Mäntymäki, M., \& Riemer, K. (2016). Enterprise social networking: A knowledge management perspective. International Journal of Information Management, 36(6), 1042-1052. http://doi.org/10.1016/j.jijinfomgt.2016.06.009

(28) Negruşa, A. L., Rus, R. V., \& Sofică, A. (2014). Innovative Tools Used by Business Networks and Clusters in Communication. Procedia - Social and Behavioral Sciences, 148, 588-595. http://doi.org/10.1016/j.sbspro.2014.07.084 
(29) Ogrean, C. (2013). Coordinates and Dynamics of the Relationships between Multinational Enterprises and Economic Development -A Theoretical Approach. Procedia Economics and Finance, 6, 319-327. http://doi.org/10.1016/S22125671(13)00146-9

(30) Oh, C. H., \& Rugman, A. M. (2012). Regional integration and the international strategies of large European firms. International Business Review, 21(3), 493507. http://doi.org/http://dx.doi.org/10.1016/j.ibusrev.2011.05.009

(31) Rick, S., Hurtado, F., Ángela, L., \& Martínez, M. (2018). Cultura clúster empresarial: Reto del mundo en el siglo XXI hacia un crecimiento empresarial Entrepreneur cluster culture: 21st century challenge of the world towards business growth. Espacios, 39. Retrieved from http://www.revistaespacios.com/ a18v39n16/a18v39n16p12.pdf

(32) Romero, D., \& Noran, O. (2015). Green virtual enterprises and their breeding environments: Engineering their sustainability as systems of systems for the circular economy. In IFAC-PapersOnLine (Vol. 28, pp. 2258-2265). http://doi. org/10.1016/j.ifacol.2015.06.424

(33) S., R. F. H. (2013). Initiative Analysis to Enter into Potential and Restricted Market: Small, Medium and Largest Enterprises Having Chance into a Virgin Market: Latin American (CELAC) and China (Practical, China Case). International Journal of Business and Management, 8(3), 103. http://doi.org/10.5539/ijbm. v8n3p103

(34) S. Rick, F. (2014). Empirical Result on Firms' Cluster Integration: Should Firms Evolve beyond Their Region? International Journal of Trade, Economics and Finance, 5(3), 204-211. http://doi.org/10.7763/IJTEF.2014.V5.372

(35) Shin, H., Lee, J. N., Kim, D., \& Rhim, H. (2015). Strategic agility of Korean small and medium enterprises and its influence on operational and firm performance. International Journal of Production Economics, 168, 181-196. http://doi. org/10.1016/j.ijpe.2015.06.015

(36) Terho, H., \& Jalkala, A. (2017). Customer reference marketing: Conceptualization, measurement and link to selling performance. Industrial Marketing Management. http://doi.org/10.1016/j.indmarman.2017.01.005 
(37) Tian, X., Wu, Y., Hou, P., Liang, S., Qu, S., Xu, M., \& Zuo, T. (2017). Environmental impact and economic assessment of secondary lead production: Comparison of main spent lead-acid battery recycling processes in China. Journal of Cleaner Production, 144, 142-148. http://doi.org/10.1016/j.jclepro.2016.12.171

(38) Uribe-Pérez, N., \& Pous, C. (2017). A novel communication system approach for a Smart City based on the human nervous system. Future Generation Computer Systems. http://doi.org/http://dx.doi.org/10.1016/j.future.2016.12.035

(39) Whiffen, T. R., Naylor, S., Hill, J., Smith, L., Callan, P. A., Gillott, M., ... Riffat, S. B. (2016). A concept review of power line communication in building energy management systems for the small to medium sized non-domestic built environment. Renewable and Sustainable Energy Reviews, 64, 618-633. http:// doi.org/10.1016/j.rser.2016.06.069

(40) Zhang, W., Zheng, C., Song, Z., Deng, A., \& He, Z. (2015). Chapter 3 Farming systems in China: Innovations for sustainable crop production. In Crop Physiology (pp. 43-64). http://doi.org/10.1016/B978-0-12-417104-6.00003-0 